\title{
Performance Evaluation of a Non- COHERENT Digital DELAY-LOCKED LOOP IN RAYLEIGH FADING CHANNELS
}

\author{
Mohamed G. El-Tarhuni \\ Department of Electrical Engineering, American University of \\ Sharjah, Sharjah, United Arab Emirates \\ mtarhunicaus.edu
}

\begin{abstract}
$P N$ code tracking is one of the most important factors that affect the performance of spread spectrum receivers. It is desirable to maintain $P N$ code tracking for a long period of time and with high accuracy. This paper presents a non-coherent digital delay-locked loop (DLL) for code tracking in direct-sequence spread spectrum (DS-SS) systems. The proposed technique utilizes three non-coherent correlators with time-diversity followed by a lock detector. Analytical results for the mean time to lose lock, also called mean hold-in time, are derived for both additive white Gaussian noise and Rayleigh fading channels using a Markov chain model. Computer simulation results are also presented to validate the analytical results. It is shown that the proposed loop can maintain lock for relatively long periods of time under practical range of signal-to-noise ratio. For instance, the loop maintains lock for more than $10^{6}$ frames for an energy per bit-to-noise power spectral density (Eb/No ) of $4 \mathrm{~dB}$ or better. It is also shown that the mean hold-in time performance is only slightly degraded due to fading because of the time diversity gain utilized by the loop in obtaining the timing information. Finally, the ability of the proposed technique to successfully track randomly and linearly varying delays is demonstrated.
\end{abstract}

\section{KEYWORDS}

PN code tracking, spread spectrum, non-coherent DLL, time-diversity, mean hold-in time

\section{INTRODUCTION}

Spread spectrum communications is considered as a leading technology for use in wideband mobile radio systems. Capitalizing on the ability of spread spectrum signals to mitigate different types of interference, such as multi-user interference and multipath interference, spread spectrum systems enjoyed worldwide adaptation for third generation $(3 \mathrm{G})$ mobile radio systems. However, such systems face the challenging task of performing code synchronization. This is very crucial since any spread spectrum based communication system requires reliable and accurate code phase timing information to de-spread the received signal and ensure satisfactory operation. This is deemed to be a very challenging task, especially over highly dispersive wireless channels. The code synchronization process is usually developed over two stages: acquisition and tracking. In this paper, we focus on the second stage of the synchronization process, i.e. code tracking.

Code acquisition is used to coarsely align the received signal and the locally generated pseudo random (PN) code to within one chip duration. This is usually accomplished by searching through the delay uncertainty window at one-chip or half-chip steps and finding the offset that would result in maximum correlation. The search process can be done using a single correlator to test one offset at a time, known as serial search, which has less complexity but long search time. On the other hand, multiple correlators could be used to test all offsets at the same time,

DOI : $10.5121 /$ ijenc.2010.2607 
known as parallel search, leading to fast search but at the cost of more complexity [1]. There are many other techniques for code acquisition including matched filter based, adaptive filter based, sequential estimation schemes, etc.

Once code acquisition is accomplished, code tracking is used to minimize the delay offset and to maintain synchronization between the signals. In most code tracking techniques known in the literature, a closed loop structure, such as the delay-locked loop (DLL) and the tau-dither loop (TDL) [1][2][1][2], is used to perform fine alignment of PN code offset and minimize the timing error. In such structures, the incoming signal is correlated either simultaneously (in DLL) or alternately (in TDL) with delayed and advanced versions of the local PN code (usually one chip or less apart), and the correlators' outputs are subtracted to generate an error signal. This signal is then used to adjust a voltage controlled clock (VCC) that derives the local PN code generator to minimize the time delay offset.

The performance of a code tracking loop is illustrated by the discriminator characteristic, usually known as the S-curve, that gives a graphical view of how the normalized tracking error is changed as the delay offset between the locally generated code and the received signal is varied. Another measure for DLL performance is the mean time to lose look, also called the mean hold-in time. This time indicates the duration over which a DLL can maintain synchronization with the received signal and it is desirable to maximize this time as much as possible. Most of the early work on DLL focused on the AWGN case but it was demonstrated that significant degradation in performance could be incurred due to other impairments such as multipath and multiple access interference (MAI) predominant in mobile radio channels [3] [4].

\section{RELATED WORK}

There has been a significant interest in developing new tracking loop algorithms to improve the performance of conventional DLL operating over many channel impairments such as multipath propagation, MAI, and time varying channel conditions. For instance, higher-order loops have been proposed to improve the tracking performance of signals delayed due to a constant relative acceleration between transmitter and receiver [5]. However, analytical evaluation of the mean time to lose lock is quite complicated and some approximations are usually required. An improved DLL structure, which uses more correlators to extend the range of delay error that can be tracked, has been investigated in [6]. The main drawback is the increased complexity and tracking jitter.

Recently, several techniques have been proposed to improve the DLL performance in fading conditions. For instance, a coherent tracking loop with maximal ratio RAKE-like structure is proposed in [7]. This scheme provides better performance compared to the non-coherent DLL assuming that accurate channel estimation is performed. However, this is generally not an easy task to achieve especially for fast varying mobile radio channels. Recognizing that a noncoherent DLL is what practically works over such hostile channel conditions, various noncoherent DLL with diversity and/or multipath interference cancellation techniques [8][9][10] and tracking algorithms based on maximum likelihood delay estimation [11], were investigated. To avoid channel estimation, a differentially coherent delay-lock loop was proposed in [12] but only the case of AWGN channel was considered. The work in [13] has presented a digital noncoherent tracking loop with RAKE-like structure to mitigate frequency selective fading. A decision-feedback coherent DLL was analyzed in [14] through the development of upper and lower bound on the tracking error over fading conditions.

A modified tracking loop that deals with MAI was presented in [15]. In this case, the authors used a linear combination of all users' code sequences as a reference signal in the DLL. The 
objective was to use a decorrelator structure to remove the effect of MAI as well as to minimize the bias term in the S-curve due to unbalances in the DLL. A simplified but suboptimal structure was presented in [16] to reduce the complexity of such algorithm.

The impact of multipath propagation on tracking loops used in Global Positioning System (GPS) receivers was investigated in many studies. For example, a model for multipath effects was developed in [17] and verified through bench tests. Both coherent and non-coherent loops were investigated with traditional early-late correlators spacing of 1 chip and a relatively narrow spacing of 0.1 chips. Recently, analytical work to assess the performance of a coherent tracking loop for positioning and navigation applications was presented in [18] and [19]. The impact of sampling and quantization on tracking loop performance, with a non integer chip-to-sample ratio, was considered in [20].

In this paper, we present a modified DLL structure using early, late, and on-time correlators to form the timing adjustment decision. We remark that there is no increase in complexity compared to conventional DLL because of the third correlator since it is already utilized for demodulation of the signal. To mitigate the effect of channel fading, the proposed scheme utilizes time diversity in forming the error signal used to track the delay. The performance measure used in this paper is the mean time to lose lock; which is also called the mean hold-in time. Furthermore, the ability of the loop to track a time varying delay is demonstrated through simulation. The paper also presents a Markov model for analyzing the tracking performance of spread spectrum systems in fading environments.

The rest of paper is organized as follows. Section 3 describes the system model and the proposed tracking algorithm. The analytical performance evaluation is presented in Section 4, and Section 5 shows simulation and numerical results. Finally, the conclusions are summarized in Section 6.

\section{PROPOSED PN CODE TRACKING ALGORITHM}

In this paper, we consider a spread spectrum system similar to the CDMA2000 system in the development of the proposed tracking look. However, the proposed scheme can be applied to other spread spectrum signals without major modification. In the considered system, during traffic mode of operation, a pilot channel is continuously transmitted by the mobile station in the reverse link along with user data. The pilot channel is utilized to achieve several tasks such as initial PN code acquisition during the access mode, acquisition of multipath timing in traffic mode, tracking of multipath components, reverse link channel estimation for coherent RAKE combining, and to assist in forward link power control.

The reverse link pilot, which is a spread spectrum signal with QPSK-spreading and no data modulation, is continuously transmitted during access and traffic modes of operation. The transmitted pilot signal is given by

$$
s_{p}=\sqrt{E_{c}}\left[\cos \omega_{c} t \sum_{k} a_{I, k} h\left(t-k T_{c}\right)+\sin \omega_{c} t \sum_{k} a_{Q, k} h\left(t-k T_{c}\right)\right]
$$

where $a_{I, k}, a_{Q, k}$ are the in-phase (I) and quadrature $(Q)$ PN code sequences, $h(t)$ is the impulse response of the transmitter's band-limiting filter assumed to follow the root-raised cosine shape with roll-off factor $r, \omega_{c}$ is the carrier frequency, and $E_{c}$ is the pilot energy per chip. The received signal over a multipath mobile radio channel may be expressed as 


$$
\begin{aligned}
r(t)= & \sqrt{E_{c}} \sum_{l=1}^{L} \alpha_{l}\left[\cos \left(\omega_{c} t-\varphi_{l}\right) \sum_{k} a_{I, k} h\left(t-k T_{c}-\tau_{l}\right)+\sin \left(\omega_{c} t-\varphi_{l}\right) \sum_{k} a_{Q, k} h\left(t-k T_{c}-\tau_{l}\right)\right] \\
& +s_{d}(t)+i(t)+n(t)
\end{aligned}
$$

where $L$ is the number of multipath components, $\alpha_{l}, \tau_{l}$, and $\varphi_{l}$ are, respectively, the amplitude, time delay, and phase of the $l^{\text {th }}$ path, $s_{d}(t)$ is the desired user spread spectrum signal, $i(t)$ is the multiple access interference, and $n(t)$ is the bandpass additive white Gaussian noise (AWGN) with zero mean and two-sided power spectral density of $N_{0} / 2$.

To recover the desired user data, the locally generated PN code at the base station must be synchronized with the incoming code. The synchronization process involves estimating the multipath time delays, $\left\{\tau_{l} ; l=1,2, \ldots L\right\}$ during the acquisition stage to within one PN chip duration $\left(T_{c}\right)$, then performing fine estimation with higher accuracy (typically delay offset less than $\pm T_{c} / 8$ ) and maintaining the codes aligned during the tracking stage. It is assumed that the acquisition algorithm has successfully acquired the timing of multipath components with enough accuracy $\left( \pm T_{c} / 2\right)$, and a tracking loop is assigned to each path to maintain synchronization.

The tracking loop should maximize the mean hold-in time; i.e. the average time it can maintain synchronization with adequate accuracy. It is also desirable to minimize the false alarm penalty time which is the time spent by the tracking loop trying to synchronize to a false delay estimate that has erroneously passed the acquisition stage. The other objective is to have a loop that minimizes the tracking error between the codes in order to maximize the signal-to-noise ratio at the demodulator output.

The proposed tracking algorithm utilizes three correlators to obtain the correlation function of the PN codes at three delays; on-time correlator, $T_{c} / 2$ - delayed correlator (late), and $T_{c} / 2$ advanced correlator (early). The on-time correlator has $I$ and $Q$ local codes with their phases set according to the delay estimate provided by the acquisition stage. The late and early correlators have their local codes one-half chip delayed and advanced from the on-time phase, respectively. A block diagram of the tracking loop is shown in Figure 1. Note that the structure shown is for one path only but the same hardware can be used to track multiple paths using different data block. The block diagram of a non-coherent QPSK correlator is shown in Figure 2.

The operation of the loop is as follows. The received signal is converted to baseband, passed through a filter matched to $h(t)$, and over-sampled such that there are $N_{s}$ samples per PN chip (for example $N_{s}=8$ ). The samples ( $I$ and $Q$ ) are then applied to the three correlators after introducing a delay offset of $\Delta$ samples where initially this offset is assumed to be zero. The signals are correlated for a period of time $N_{c}$ (typically $N_{c}=256$ PN chips) and, to improve the signal-to-noise ratio and reduce the effect of fading and time variations in the channel, the outputs of the correlators are non-coherently accumulated (added) over $n$ non adjacent correlations or blocks of data to yield $Z_{e}, Z_{l}$ and $Z_{o}$ for the early, late, and on-time correlators, respectively, as shown in Figure 1. These results are used to update the tracking loop delay estimate to minimize the tracking error and maintain synchronization. The required offset $\Delta$ in samples is computed from the difference of the early and late correlations normalized by the ontime correlator output such that $\Delta=\left(Z_{e}-Z_{l}\right) / Z_{o}$, and the input signal timing is adjusted $\Delta$ samples (either advanced or delayed) to retain the symmetry of the correlation results around the 
centre value. The tracking loop is updated fast enough to ensure that changing delays are tracked with high accuracy.

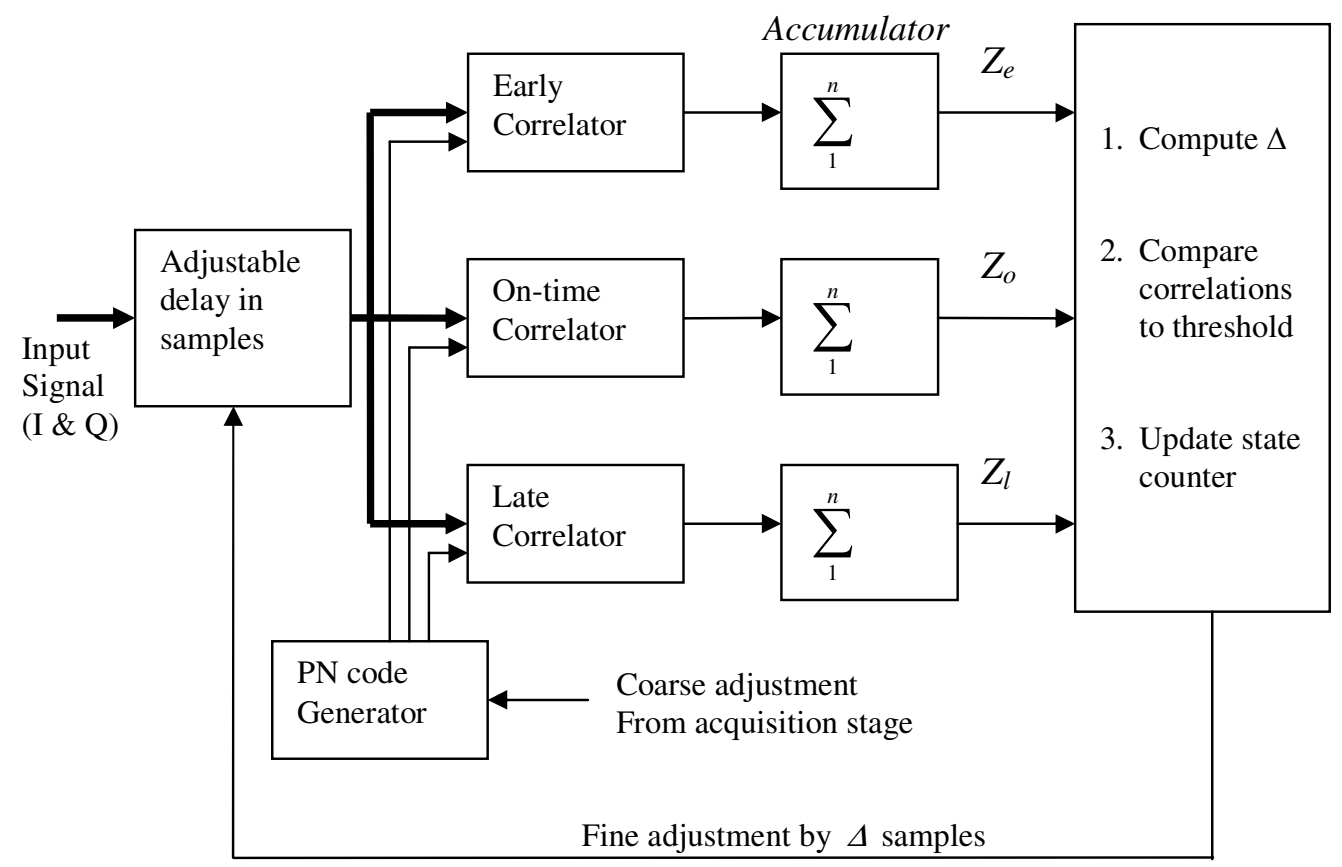

Figure 1. Block diagram of the proposed tracking loop.

The ability of the proposed scheme to compensate for the timing error is illustrated by the $S$ curve shown in Figure 3. Both the conventional DLL with no division with the on-time correlator and the proposed DLL with division by $Z_{o}$ are shown for a raised cosine pulse with roll-off factor $r=1$ and $r=0.22$. It is clear that the proposed scheme extends the lock-in range to more than $\pm T_{c} / 2$, which is the tracking range obtained by the conventional scheme. As we can see, the $S$-curve for the proposed scheme continuous to increase as the timing error exceeds the half-chip range while the conventional DLL $S$-curve starts to decrease indicating the start of losing lock. In fact, when the timing error reaches one-chip, the proposed DLL will have an Scurve that goes to infinite because of the division by zero since the autocorrelation function of the raised-cosine pulse will reach zero at that offset. In practice this is not going to cause any problem since we can limit the maximum value of the $S$-curve to a certain limit and after all we should not expect the offset to reach the one-chip value since the loop is supposed to maintain it within half-chip range. In case the offset is noticed to increase beyond half-chip, the local PN code offset may be coarsely adjusted by half-chip to compensate for that error.

The decision regarding the state of the tracking loop; i.e. in-lock or out-of-lock; is made according to the state of an up/down counter. The counter is initially set at state " 0 " and it is state is updated at the same time the tracking loop is updated. If the any of the correlators outputs, $Z_{e}, Z_{l}$ or $Z_{o}$, exceeds a fixed threshold $(\eta)$ then the counter is reduced by one, with a minimum count zero. On the other hand, if none of the correlators' outputs exceeds the threshold, indicating a possible loss of lock, then the counter state is increased by one. Once the counter state reaches a predetermined value, e.g. a value of 3 , the loop declares an out-of-lock condition; and a new acquisition phase is started. 


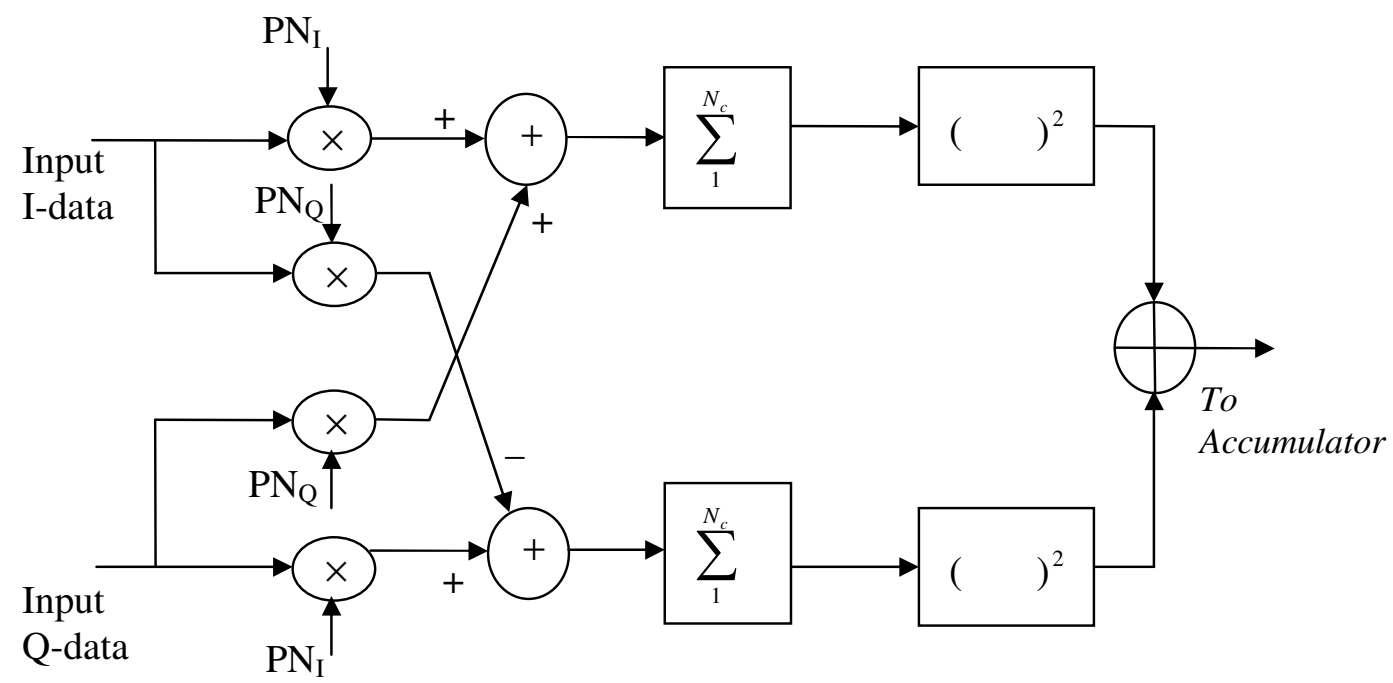

Figure 2. Block diagram of non-coherent correlator.

\section{PERFORMANCE EVALUATION}

As mentioned previously, the lock detector is an up/down counter used to monitor the status of the tracking process in a fashion similar to the Search/Lock Strategy (SLS) described in [21][22]. The tracking process is best described by a finite state Markov chain as depicted in Figure 4. The counter is initialized to zero, and after a period of time, $T_{u}$ (update period), the counter is either incremented to one with probability $1-P_{D}$ (i.e. all correlators are less than the threshold) or it stays at zero with probability $P_{D}$ (i.e. one or more of the correlators is greater than the threshold). Similarly, if the counter state is one, it may be incremented after a period of $T_{u}$ to two or decremented to zero with probability $1-P_{D}$ or $P_{D}$, respectively. Eventually, the counter will reach three (the absorbing state) which represents the out-of-lock condition. Absorption time is defined as the time required for the Markov chain to reach the absorbing state 3 from any transient state 0,1 , or 2 . Starting from state 0 , absorption time is interpreted as the time to lose lock or the time to clear a false alarm depending upon what has caused the initiation of the tracking mode. For instance, if a true detection triggered the loop to track a correct path delay, then the time for the counter to reach the absorbing state 3 is the time to lose lock of the loop. On the other hand, if what actually triggered the loop was a false alarm with a wrong delay, the absorption time is the false alarm penalty time.

The Markov chain is analyzed by defining its state transition matrix. This matrix is a square matrix whose $k l^{\text {th }}$ element represents the probability of going from state $k$ to state $l$ in one step. It is beneficial to arrange the transition matrix into its canonical form as defined in [22]. For the system under consideration, the canonical form is given by 
International Journal of Computer Networks \& Communications (IJCNC) Vol.2, No.6, November 2010



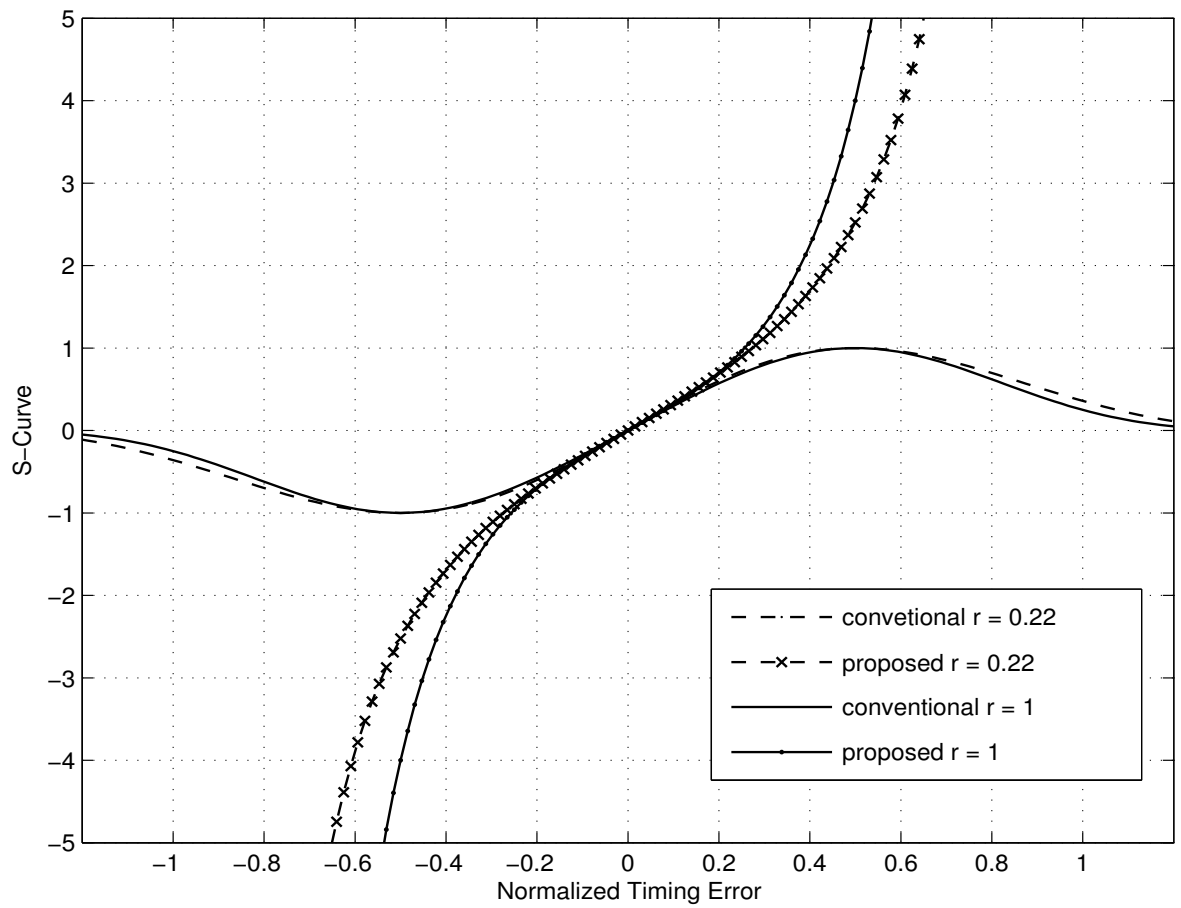

Figure 3. $S$-Curve for the proposed DLL and conventional DLL with raised-cosine pulse.

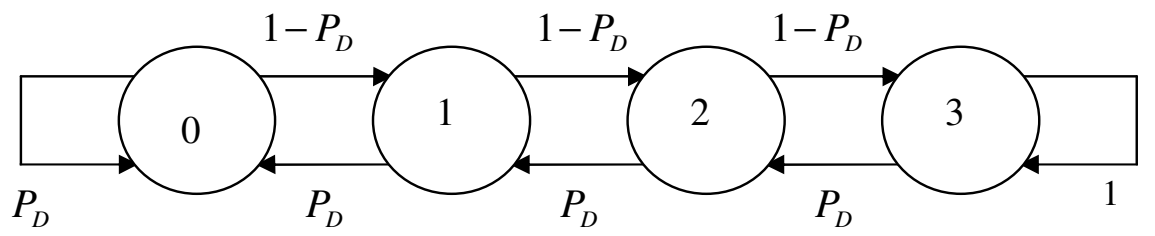

Out-of-lock state

Figure 4. Flow graph for the 3-state up/down counter.

where $\beta$ is an identity matrix representing the absorbing states, $\Psi$ is a matrix of all zeros, $Q$ contains the transition probabilities between transient states, and $\Gamma$ gives the transition probabilities from transient states to absorbing states. The mean absorption time is given by the vector [21] 
International Journal of Computer Networks \& Communications (IJCNC) Vol.2, No.6, November 2010

$$
\bar{T}_{a b s}=\left[\begin{array}{c}
\bar{T}_{03} \\
\bar{T}_{13} \\
\bar{T}_{23}
\end{array}\right]=[I-Q]^{-1} \bar{T}
$$

where $I$ is the identity matrix with same dimensions as $Q$ and $\bar{T}$ is a vector representing the transition times between states. In the system at hand, all elements of $\bar{T}$ equal to $T_{u}$. Using (3) into (4), the mean time for the counter to reach a value of three starting from a value of zero can be shown to be [23]

$$
T_{L L}=\frac{1+2\left(1-P_{D}\right)^{2}}{\left(1-P_{D}\right)^{3}} T_{u}
$$

where $T_{u}$ is the tracking loop update time, and, as defined earlier, $P_{D}$ is the probability that one or more of the correlators exceed a preset threshold, $\eta$. Since the offset between the correlators is one-half chip and the auto-correlation functions extends from $\left(-T_{c}\right.$ to $\left.T_{c}\right)$, two or all of the three correlations may correspond to the in-phase hypothesis $H_{1}$. Therefore, $P_{D}$ may be expressed as

$$
P_{D}=1-\prod_{j=1}^{3}\left(1-P_{j}\right)
$$

with $P_{j}$ defined as the probability that the $j^{\text {th }}$ correlator output is below the threshold $\eta$, which is calculated as

$$
P_{j}=\int_{\eta}^{\infty} f_{j}(x) d x, \quad j=1,2,3
$$

where $f_{j}(x)$ is the probability density function (PDF) of the $j^{\text {th }}$ correlator output given by [24]

$$
f_{j}(x)=\left(\frac{x}{n R_{j}^{2} \mu}\right)^{\frac{n-1}{2}} e^{-\left(x+n R_{j}^{2} \mu\right)} I_{n-1}\left(2 \sqrt{n R_{j}^{2} \mu x}\right), \quad x \geq 0
$$

for AWGN case, and

$$
f_{j}(x)=\frac{x^{n-1}}{(n-1) !} \frac{e^{-\left(\frac{x}{1+R_{j}^{2} \bar{\mu}}\right)}}{\left(1+R_{j}^{2} \bar{\mu}\right)^{n}}, \quad x \geq 0
$$

for Rayleigh fading channels. The parameters in (8) and (9) are defined as follows:

$n$ - Number of non-coherent accumulations of each correlator

$R_{j}$ - Autocorrelation function of $h(t)$ at the $j^{t h}$ correlator

$\mu$ - Average signal-to-noise ratio per correlation period of $N_{c}$ chips $\left(\mu=N_{c} E_{c} / N_{0}\right)$

$\bar{\mu}$ - Average signal-to-noise ratio with Rayleigh fading $\left(\bar{\mu}=E\left[\alpha^{2}\right] \mu\right)$

$I_{n}-n^{\text {th }}$ Order modified Bessel function of the first kind 
The normalized threshold $\eta$ is evaluated according to the desired probability of false alarm of the tracking loop. This false alarm occurs when the acquisition stage passes a wrong delay estimate to the tracking loop and the loop tries to lock into this false signal. It is desirable to minimize the time wasted by the loop in recognizing that a false alarm had occurred, called mean false alarm penalty time. We remark that if the threshold is set to a small value, then the loop may spend a very long time to dismiss the wrong delay. On the other hand, if the threshold is set to a large value, the loop can rapidly detect the false alarm errors but it may tend to rapidly lose lock to correct delays. Therefore the threshold should be set to compromise these effects.

The false alarm probability is given by

$$
P_{F A}=\int_{\eta}^{\infty} f_{H 0}(x) d x
$$

where $f_{H 0}(x)$ is the PDF of the decision statistic, $Z_{o}$, under hypothesis $H_{0}$ where no signal is present and is given by [24]

$$
f_{H 0}(x)=\frac{x^{n-1}}{(n-1) !} e^{-x}, \quad x \geq 0
$$

We remark that, for a given false alarm probability, the threshold $\eta$ can be obtained from (10). However, the value obtained is actually normalized by the variance of the noise at the output of the correlator.

\section{NUMERICAL AND SIMULATION RESULTS}

Computer simulations and numerical evaluation of the performance and functionality of the proposed tracking loop have been performed. The chip rate of the system is set to $1.2288 \times 10^{6}$ chips per second and the frame duration is $20 \mathrm{~ms}$; as per CDMA2000 standard with Spreading Rate 1 . The delay offset between the correlators is $T_{c} / 2$, the number of samples per chip is set to $N_{s}=8$, the correlation period is $N_{c}=256 \mathrm{chips}$, and the number of accumulations is $n=6$ or $n=12$. Note that for a $20 \mathrm{~ms}$ frame, there are 96 blocks each of length 256 chips. Thus, with $n=6$ accumulations, the following blocks are used for combining: 1, 17, 33, 49, 69 and 81. For $n=12$, the blocks used for accumulations are: 1, 9, 17, 25, 33, 41, 49, 57, 65, 73, 81 and 89 . The spacing of the blocks used for accumulation is basically used to ensure that the data used is uncorrelated so that the effect of channel fading is minimized (time diversity). We remark that the system should work properly for any other combinations as long the blocks are spaced as wide as possible. The hold-in time is normalized with respect to the update rate of the tracking

loop. As mentioned in section 3, one or more updates per frame are needed for the loop to follow the delay variations. For a false alarm probability of 0.1 , the normalized thresholds as computed from (10) are 9.27 and 16.6 for $n=6$ and $n=12$ accumulations, respectively. Finally, we note that the parameters set in this section are typical values and would not be considered as a best case for the proposed loop. The main task is to see how the proposed loop would perform under typical channel conditions. 
The ability of the loop to track time varying delays is investigated using simulation. Two cases for the tracking behaviour of the loop in AWGN with signal-to-noise ratio per chip, $S N R_{c}$ of $-20 \mathrm{~dB}$ are presented. The first scenario, as shown in Figure 5, is tracking of a delay that is varying according to a random walk process with the delay either increased or decreased by one sample with equal probability. The second scenario is a linearly increasing delay with a delay change rate of one sample per frame as shown in Figure 6. We observe that the tracking loop successfully tracks the varying delays with high accuracy. There are few instants where the loop is offset from the actual delay by one or two samples, but it recovers rapidly to the correct delay.

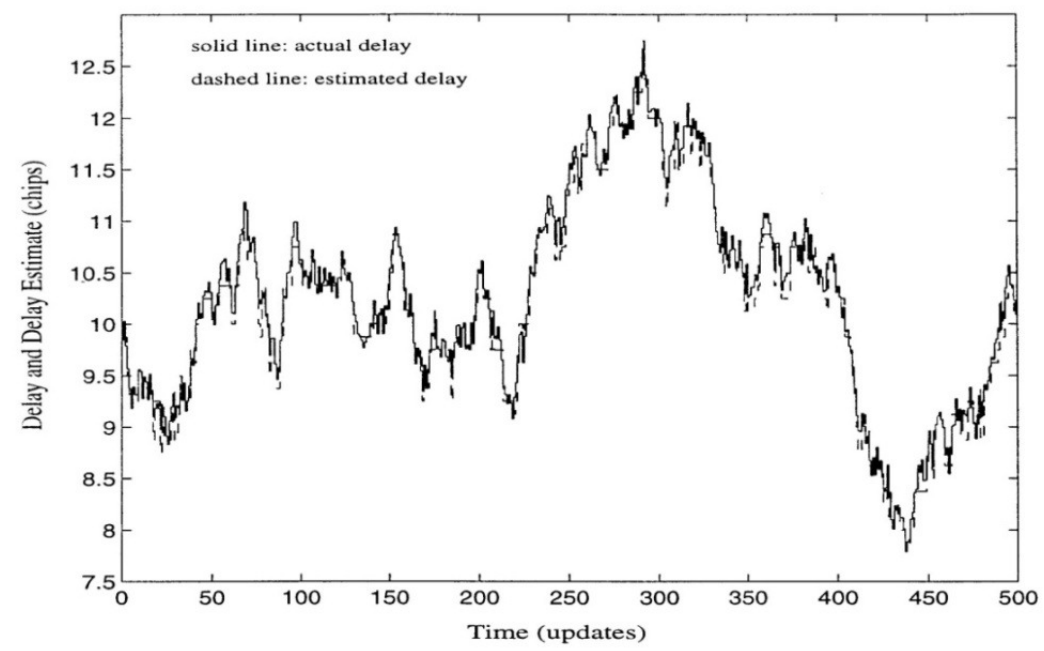

Figure 5. Tracking of randomly varying delay.



Figure 6. Tracking of linearly varying delay. 
The mean hold-in time of the tracking loop is evaluated over AWGN and fading channels. Figure 7 shows the mean-hold in time verses the $S N R_{c}$ in AWGN. As we can see, with the loop updated once every frame; the loop can keep track of the incoming signal at $S N R_{c}$ of $-26 \mathrm{~dB}$ for about 60 and 400 frames with 6 and 12 accumulations, respectively. Simulation results are in good agreement with the analytical results. Thus doubling the number of accumulations results in significant increase in the hold-in time.

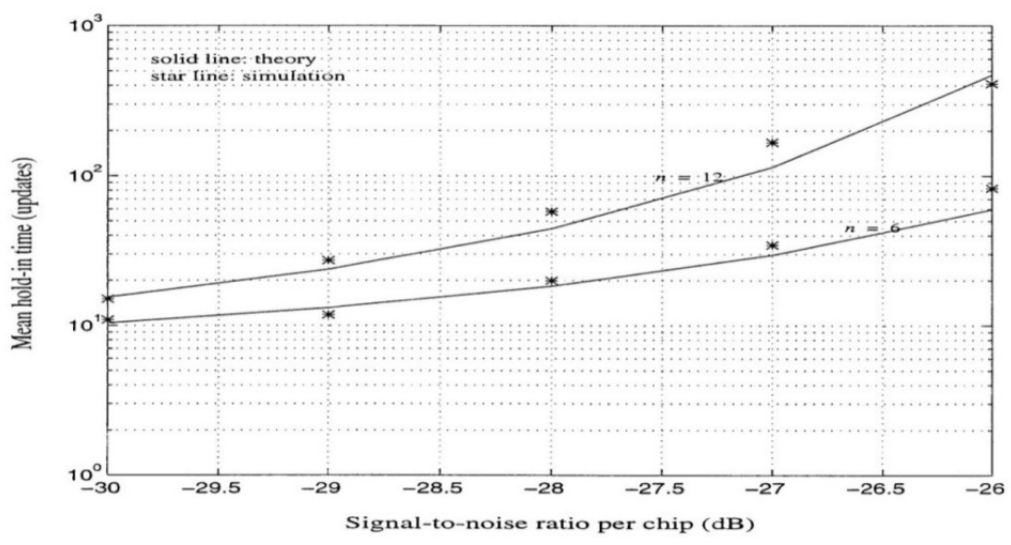

Figure 7. Mean-hold-in time in AWGN.

Analytical results indicate that the mean hold-in time increases exponentially with $S N R_{c}$. For instance, Figure 8 shows that, when $S N R_{c}$ is increased above $-21 \mathrm{~dB}$, the mean hold-in time is increased to more than $10^{6}$ frames. Simulations are not available to verify these analytical results because of the very long run time needed. However, since the simulations agree with the theory in the low range of $S N R_{C}$, we have more confidence in the analytical results and can use them as an indicator of the excellent performance of the loop.

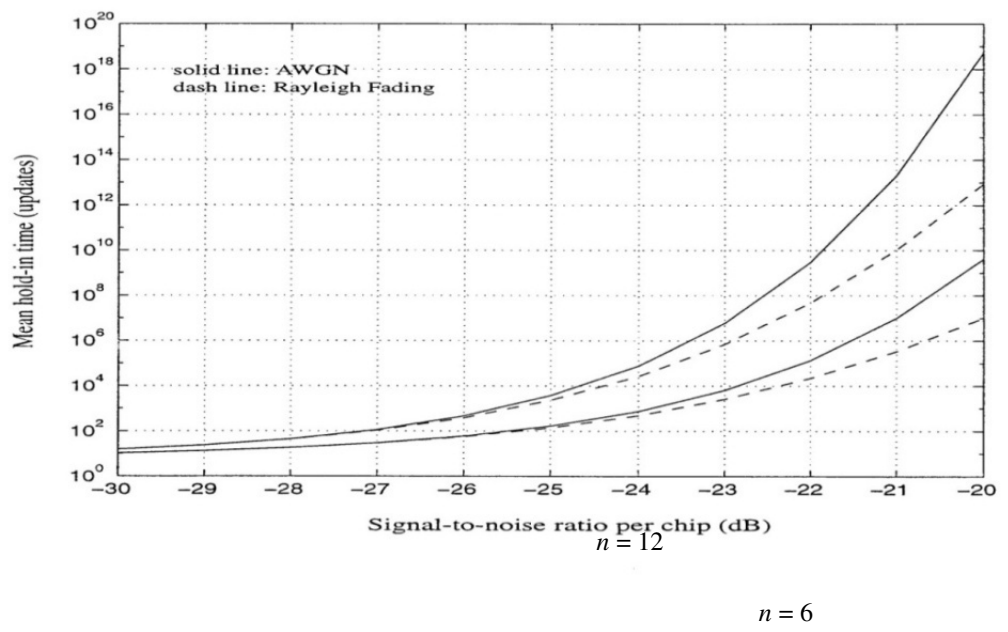

Figure 8. Analytical evaluation of mean hold-in time for large SNR with $n=6$ and $n=12$. 
The performance in Rayleigh fading channel with a normalized Doppler spread of $10^{-3}$ is shown in Figure 9. The results show that fading does not degrade the performance significantly and the loop can maintain lock for all practical range of $S N R_{c}$. The loss is less than one $\mathrm{dB}$ compared to AWGN case. This is attributed to the time diversity gain obtained by using non adjacent data blocks to obtain the correlation results and hence average out the effect of fading. It is also attributed to the lock detector used since even when the correlation results are degraded the up/down counter gives another chance to recover when the channel conditions gets better. We remark that a lock detector with a number of states different than three could have been used. Increasing the number of states would increase the mean hold-in time but at the same time would increase the false alarm penalty time. On the other hand, reducing the number of states would result in short false alarm penalty time but with a reduction in the mean hold-in time.

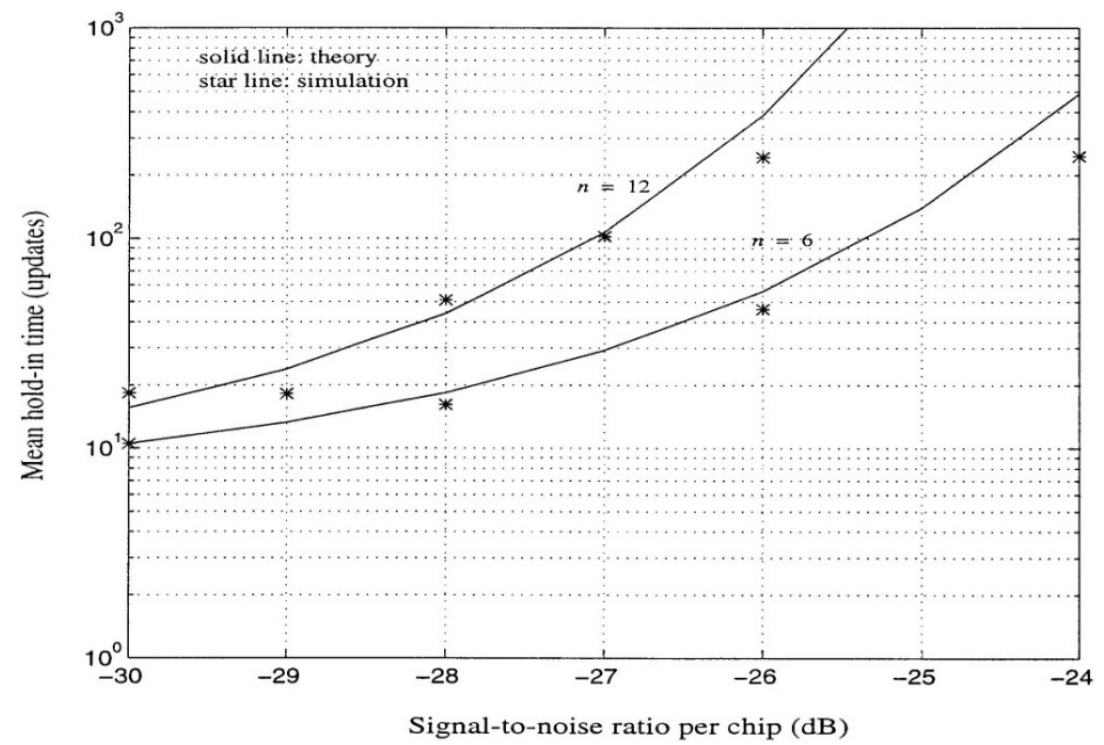

Figure 9. Mean hold-in-time in Rayleigh fading.

\section{Conclusions}

A PN code tracking loop using a digital DLL structure has been analyzed for application in spread spectrum signals. The tracking loop is based on using three non-coherent correlators and phase-shifting of input signal. The timing error is obtained from the three correlation results to improve the accuracy of delay estimation with no increase in complexity. The mean hold-in time performance of the proposed loop is evaluated analytically and using simulation over both AWGN and Rayleigh fading channels. A finite state Markov chain model is used in developing the analytical performance. The accuracy of the analytical evaluation was validated through Monte Carlo simulations. The performance results indicate that the loop provides excellent hold-in time performance and can track varying delays accurately. The proposed structure uses non adjacent data blocks of the received signal and an up/down lock detector that results in an improved performance against fading. 


\section{REFERENCES}

[1] M. Simon, J. Omura, R. Scholtz, B. Levitt, Spread Spectrum Communications Handbook, McGraw-Hill, Inc., 1994.

[2] A. Polydros and C. Weber, "Analysis and optimization of correlative code-tracking loops in spread-spectrum systems," IEEE Trans. Commun., vol. 33, no. 1, pp. 331-339, Jan. 1985.

[3] W. Braun, "PN acquisition and tracking performance in DS/CDMA systems with symbollength spreading sequences," IEEE Trans. Commun., vol. 45, no. 12, pp. 1595-1601, Dec. 1997.

[4] J. Caffery Jr. and G. Stuber, "Effects of multiple-access interference on the noncoherent delay lock loop,” IEEE Trans. Commun., vol. 48, no. 12, pp. 2109-2119, Dec. 2000.

[5] A. Welti, U. Bernhard, and B. Bobrovsky, "Third-order delay-locked loop: mean time to lose lock and optimal parameters," IEEE Trans. Commun., vol. 43, no. 9, pp. 2540-2550, Sept. 1995.

[6] A. Wilde, "The Generalized Delay-Locked Loop," Wireless Personal Communications Journal, vol. 8, no. 2, pp. 113-130, Sept. 1998.

[7] W. Sheen and G. Stuber, "A new tracking loop for direct sequence spread spectrum systems on frequency-selective fading channels," IEEE Trans. Commun., vol. 43, no. 12, pp. 3063-3072, Dec. 1995.

[8] W. Sheen and C. Tai, "A non-coherent tracking loop with diversity and multipath interference cancellation for direct-sequence spread-spectrum systems," IEEE Trans. Commun., vol. 46, no. 11, pp. 1516-1524, Nov. 1998.

[9] H. Boujemaa and M. Siala, "Enhanced coherent delay tracking for direct sequence spread spectrum systems," in Proc. $6^{\text {th }}$ International Symposium on Spread Spectrum Techniques \& Applications, 2000, pp. 247-277.

[10] G. Fock, J. Baltersee, P. Schlz-Rittich, and H. Meyr, "Channel tracking for Rake receivers in closely spaced multipath environments," IEEE J. Select. Areas Commun., vol. 19, no. 12, pp. 2420-2431, Dec. 2001.

[11] E. Sourour, G. Bottomley, and R. Ramesh, "Delay tracking for direct sequence spread spectrum systems in multipath fading channels," in Proc. $49^{\text {th }}$ IEEE Vehicular Technology Conference, 1999, pp. 422-426.

[12] C. Fan and Z. Tsai, "A differentially coherent delay-locked loop for spread-spectrum tracking receivers,” IEEE Commun. Letters, vol. 3, pp. 282-284, 1999.

[13] J. Lin, "A modified PN code tracking loop for direct-sequence spread spectrum communication over arbitrarily correlated multipath fading channels," IEEE J. Select. Areas Commun., vol. 19, no. 12, pp. 2381-2395, Dec. 2001.

[14] W. Wong, Y. Wu, S. Leung, and Y. Zhu, "A modified decorrelated delay lock loop for synchronous DS-CDMA systems," IEEE Trans. Veh. Technol., vol. 57, no. 5, pp. 3293-3299, Sep. 2008.

[15] Hyung-Rae Park, "Performance analysis of a decision-feedback coherent code tracking loop for pilot-symbol-aided DS/SS systems," IEEE Trans. Veh. Technol., vol. 55, no. 4, pp. 1249 1258, July 2006.

[16] Y. Wu, S. Leung, W. Wong, and Y. Zhu, "A suboptimal modified code tracking loop for synchronous DS-CDMA systems," in Proc. $60^{\text {th }}$ IEEE Vehicular Technology Conference, 2009.

[17] S. Kalyanaraman, M. Braasch, and J. Kelly, "Code tracking architecture influence on GPS carrier multipath," IEEE Trans. Aerospace and Electronic Systems, vol. 42, no. 2, pp. 548 561, April 2006. 
[18] J. Betz and K. Kolodziejski, "Generalized theory of code tracking with an early-late discriminator. Part I: Lower bound and coherent processing," IEEE Trans. Aerospace and Electronic Systems, vol. 45 , no. 4, pp. 1538 - 1556, Oct. 2009.

[19] J. Betz and K. Kolodziejski, "Generalized theory of code tracking with an early-late discriminator. Part II: Noncoherent processing and numerical results," IEEE Trans. Aerospace and Electronic Systems, vol. 45 , no. 4, pp. 1557-1564, Oct. 2009.

[20] M. Srinivasan and K. Quirk, "PN Code tracking using noncommensurate sampling," IEEE Trans. Commun., vol. 54 , no. 7, pp. 1349 - 1349, Oct. 2006.

[21] P. Hopkins, "A unified analysis of pseudonoise synchronization by envelope correlation," IEEE Trans. Commun., vol. COM-25, no. 8, pp. 770-778, Aug. 1977.

[22] J. Holmes, Coherent Spread Spectrum System, Robert Krieger Publishing Company, Malabor, Florida, 1990.

[23] M. El-Tarhuni, A. Sheikh, "Performance analysis for an adaptive filter code-tracking technique in direct-sequence spread-spectrum systems," IEEE Trans. Commun., vol. 46, no. 8, pp. 10581064, Aug. 1998.

[24] A. Viterbi, CDMA - Principles of Spread Spectrum Communications, Addison Wesley, 1995.

\section{Authors}

Mohamed El-Tarhuni received the B.Sc. and M.Sc. degrees in electrical engineering from Garyounis University, Benghazi, Libya, in 1986 and 1990, respectively, and the Ph.D. degree in electrical engineering from Carleton University, Ottawa, ON, Canada, in 1997. From 1987 to 1993, he was with the General Electric Company of Libya, Benghazi, Libya, first as a Telecommunications Engineer and then as a Manager of the Communications Department. From 1997 to 2000, he was with Nortel Networks, Ottawa, ON, Canada, as a member of scientific Staff. Since 2000, he has been with the

American University of Sharjah (AUS), Sharjah, United Arab Emirates (UAE), where he is currently an Associate Professor and the Head of the Department of Electrical Engineering. From 2001 to 2006, he was the Director for the Cisco Regional Networking Academy, AUS. His current research interests include wireless and mobile radio systems, cognitive radio systems, CDMA, OFDM, physical layer issues, and cross-layer design.

Dr. El-Tarhuni has served on the organizing and technical committees of many international conferences. He also served as the IEEE Industry Relations Officer for the UAE Section from 2002 to 2006.

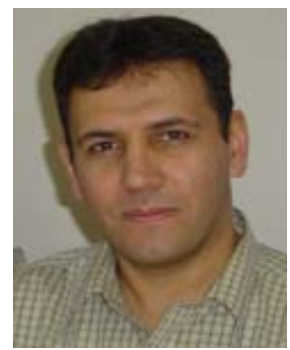

